\title{
Existence of the non-constant steady states to a fractional diffusion predator-prey system including Holling type-II functional response
}

\section{Chenglin Li* (D)}

"Correspondence:

chenglinli988@163.com School of Mathematics, Honghe University, Mengzi, Yunnan 661199, P.R. China

\section{严 Springer}

\begin{abstract}
This paper is concerned with a fractional diffusion predator-prey system with Holling type-II functional response in a bounded domain with no flux boundary condition. The local and global stabilities are investigated and sufficient conditions of stabilities are obtained. The existence of the non-constant steady states is considered and the sufficient conditions of the existence for the non-constant steady states are also obtained. The results show that the predator and the prey can coexist under some suitable conditions with fractional diffusion.
\end{abstract}

Keywords: fractional diffusion; stability; steady states

\section{Introduction}

The population always move from the higher concentration to the lower concentration by spatial heterogeneity of species. This process is often regarded as Brownian motion, which is sometimes called Gaussian diffusion or normal diffusion, and this process can be described by employing the Laplacian operator. Recently, there have been some papers in being able to reveal the dynamics of the systems with Gaussian diffusion, such as stationary patterns [1-4], globally asymptotic stability $[2,5]$ and so on. The well-known predatorprey model with normal diffusion incorporating Holling type-II functional response is as follows:

$$
\begin{aligned}
& \frac{\partial u}{\partial t}-d_{1} \Delta u=-a u+e c u v /(1+b v) \quad \text { in }(0, T) \times \Omega, \\
& \frac{\partial v}{\partial t}-d_{2} \Delta v=r(1-v / K) v-c u v /(1+b v) \quad \text { in }(0, T) \times \Omega, \\
& \frac{\partial u}{\partial v}=\frac{\partial v}{\partial v}=0 \quad \text { on }(0, T) \times \partial \Omega, \\
& (u(0, x), v(0, x))=\left(u_{0}(x), v_{0}(x)\right) \geq(0,0) \quad \text { in } \Omega,
\end{aligned}
$$

where $\Omega$ is a bounded domain in $R^{N}$ ( $N \geq 3$ is an integer) with boundary $\partial \Omega$; $u$ and $v$ stand for the densities of the predator and the prey, respectively; the positive constants $a$,

(c) The Author(s) 2017. This article is distributed under the terms of the Creative Commons Attribution 4.0 International License (http://creativecommons.org/licenses/by/4.0/), which permits unrestricted use, distribution, and reproduction in any medium, provided you give appropriate credit to the original author(s) and the source, provide a link to the Creative Commons license, and indicate if changes were made. 
$K, r, e, 1 / c, b / c$ and $1 / b$ are the death rate of the predator, the carrying capacity of the prey, the prey intrinsic growth rate, the conversion rate, the time spent by a predator to catch a prey, the manipulation time which is a saturation effect for large densities of prey, the density of prey necessary to achieve one half the rate, respectively.

However, Brownian motion is only a special Lévy process and diffusion process of animals is always intricate, for instance, animals always run into some obstacles or stay etc. As a result, the movement of animals cannot often obey the rule of normal diffusion, but it obeys Lévy diffusion which is described by a fractional Laplacian operator. Furthermore, the recent research [6] has showed that the diffusion of some animals (such as sea turtles, bony fishes, sharks and penguins) represents Lévy-walk-like behaviors. Therefore, based on this fact, we shall consider (1.1) including Lévy diffusion (fractional diffusion) instead of Gaussian diffusion. Thereinto, this diffusion has been successfully used in the epidemic model [7] and quantum physics [8, 9]. $(-\Delta)^{\alpha}$ is a corresponding infinitesimal generator which is a fractional Laplacian operator, and this fractional diffusion generated by $(-\Delta)^{\alpha}$ describes a pure jump process and is an anomalous diffusion. Moreover, its corresponding characteristic is given by $(0,0, \varsigma)[10]$, where $\varsigma$ is a Lévy measure, i.e., $\varsigma(d x)=\frac{K(s) d x}{|x|^{N+s}}$ for some positive constant $K(s)$.

Based on the fact above, system (1.1) equipped with fractional diffusion can be written as follows:

$$
\begin{aligned}
& \frac{\partial u}{\partial t}+(-\Delta)^{\alpha} u=-a u+e c u v /(1+b v) \quad \text { in }(0, T) \times \Omega, \\
& \frac{\partial v}{\partial t}+(-\Delta)^{\alpha} v=r(1-v / K) v-c u v /(1+b v) \quad \text { in }(0, T) \times \Omega, \\
& \frac{\partial u}{\partial v}=\frac{\partial v}{\partial v}=0 \quad \text { on }(0, T) \times \partial \Omega, \\
& (u(0, x), v(0, x))=\left(u_{0}(x), v_{0}(x)\right) \geq(0,0) \quad \text { in } \Omega,
\end{aligned}
$$

where $\alpha \in(0,1) ; \Omega$ is a bounded convex regular extension domain in $R^{N}(N \geq 3$ is an integer) with boundary $\partial \Omega$. Obviously, system (1.2) is an extension of model (1.1). To our knowledge, system (1.2) has not been investigated before.

The topic of interest for us is whether the various species can coexist in a predator-prey system. Sometimes, the species coexist in a steady state. The coexistence of population in the space of homogeneous distribution would be indicated by constant steady states to the system. In the spatially inhomogeneous case, it is an indication of the richness of the corresponding partial differential equational dynamics. Many authors have established the existence of non-constant steady states in normal diffusion population models [3, 1114]. The main objective of this paper is to investigate the existence of non-constant steady states which arises from the fractional diffusion system (1.2). According to the result of Theorem 5.3 in this paper, there exist non-constant steady states for system (1.2) under some suitable conditions, that is, the prey and the predator can coexist for a long period of time under some conditions.

This paper is organized as follows. In Section 2, the preliminaries are introduced. In Section 3, the existence of positive solution of (1.2) is investigated. In Section 4, the stability of positive constant equilibrium is considered. In Section 5, the existence of non-constant positive equilibrium is investigated. 


\section{Preliminaries}

Definition 2.1 ([15]) Let $\widetilde{D}$ be a bounded domain and $f: \widetilde{D} \rightarrow \mathbf{R}, x \rightarrow f(x)$, be a continuous (but not necessarily differentiable) function, and suppose that $h>0$ is a constant discretization span. Define the translation operator

$$
F W(h) f(x):=f(x+h)
$$

Then the fractional difference of order $\alpha, \alpha \in \mathbf{R}, 0<\alpha \leq 1$, of $f(x)$ is defined by the expression

$$
\Delta^{\alpha} f(x):=(F W-I)^{\alpha} f(x)=\sum_{k=0}^{\infty}(-1)^{k}\left(\begin{array}{l}
\alpha \\
k
\end{array}\right) f(x+(\alpha-k) h),
$$

where $I$ is an identity operator.

The eigenvalue problem of the fractional Laplacian operator $(-\Delta)^{\alpha}, \alpha \in(0,1)$ is given by

$$
\begin{aligned}
& (-\Delta)^{\alpha} \varphi_{k}=\mu_{k}^{\alpha} \varphi_{k}, \quad x \in \Omega, \\
& \frac{\partial \varphi_{k}}{\partial \nu}=0, \quad x \in \partial \Omega,
\end{aligned}
$$

with the domain of definition of $(-\Delta)^{\alpha}$

$$
D\left[(-\Delta)^{\alpha}\right]=\left\{w \in L^{2}(\Omega):\left\|(-\Delta)^{\alpha} w\right\|_{L^{2}(\Omega)}^{2}=\sum_{k=1}^{\infty}\left|\mu_{k}^{\alpha}\left\langle w, \varphi_{k}\right\rangle\right|^{2}<\infty, \frac{\partial w}{\partial v}=0\right\},
$$

where $\mu_{k}^{\alpha}$ is the corresponding eigenvalue of $\varphi_{k} ; \varphi_{k}$ is the corresponding eigenfunction of $(-\Delta)^{\alpha}$ and $\left\{\varphi_{k}\right\}_{k=1}^{\infty}$ is an orthonormal basis of $L^{2}(\Omega) ; 0=\mu_{1}^{\alpha}<\mu_{2}^{\alpha}<\cdots<\mu_{k}^{\alpha} \rightarrow+\infty$.

The formula of $(-\Delta)^{\alpha} u$ for $u \in C_{c}^{\infty}(\Omega)[16]$ is also given by

$$
(-\Delta)^{\alpha} u:=\text { p.v. } \int_{\Omega} \frac{u(x)-u(y)}{|x-y|^{N+2 \alpha}} d y, \quad x \in \Omega .
$$

We shall employ the following inequalities of Strook and Varopoulos (Theorem 1 in [17]):

$$
\begin{aligned}
& \int_{\Omega} u(x)(-\Delta)^{\alpha} u(x) d x \geq 0, \quad u \in D\left((-\Delta)^{\alpha}\right), \\
& \int_{\Omega} v(x)(-\Delta)^{\alpha} u(x) d x=\int_{\Omega}(-\Delta)^{\alpha / 2} v(x)(-\Delta)^{\alpha / 2} u(x) d x .
\end{aligned}
$$

In the next discussion, the following embedding theorem will be useful.

Lemma 2.1 ([16]) Assume that $\alpha \in(0,1), p \in[1, \infty)$ and $s p<N$. Let $q \in\left[1, p^{*}\right), \Omega \subset R^{N}$ be a bounded extension domain for $W^{\alpha, p}$, then the embedding $W^{s, p}(\Omega) \hookrightarrow L^{q}(\Omega)$ is compact.

Suppose that $A$ is the realization of $(-\Delta)^{\alpha}$ under the homogenous Neumann boundary condition in $L^{2}(\Omega)$. Then $-A$ is a sectorial operator and an infinitesimal generator of analytic semigroups $\left\{e^{-t A}\right\}_{t \geq 0}$. 
For convenience, we denote $x_{+}:=\max \{0, x\}, x_{-}:=\max \{0,-x\}$ for $x, y \in R$ and extend these notations to real-valued functions. If $(V, \geq)$ is a partially ordered vector space, we denote by its positive cone $V_{+}:=\{v \in V: v \geq 0\}$. Set $X=\left\{L^{2}(\Omega)\right\}^{2}$. Assume $\mathbf{w}=(u, v) \in X$, which is equipped with the norm $\|\mathbf{w}\|=\|u\|_{2}+\|v\|_{2}$.

\section{Existence of positive solution of (1.2)}

In this section, we shall prove the existence of local mild solution for system (1.2). Let $f_{1}(u, v)=-a u+e c u v /(1+b v)$ and $f_{2}(u, v)=r(1-v / K) v-c u v /(1+b v)$.

Definition 3.1 Let $\left(u_{0}, v_{0}\right) \in X$ and $T>0 .(u, v) \in C([0, T] ; X)$ is called a mild solution of (1.2) if $(u, v)$ satisfies

$$
\begin{array}{ll}
u(t)=e^{-t A} u_{0}+\int_{0}^{t} e^{-(t-s) A} f_{1}(u(s), v(s)) d s, & t \in[0, T], \\
v(t)=e^{-t A} v_{0}+\int_{0}^{t} e^{-(t-s) A} f_{2}(u(s), v(s)) d s, & t \in[0, T] .
\end{array}
$$

Then system (1.2) can be rewritten as the following equation:

$$
\begin{aligned}
& \frac{d z}{d t}=Q z+F(z), \quad t \in(0,+\infty), \\
& z(0)=z_{0}
\end{aligned}
$$

where $z=(u(t, x), v(t, x))^{T}, z_{0}=\left(u_{0}, v_{0}\right)^{T}$,

$$
Q=\left(\begin{array}{cc}
-A-1 & 0 \\
0 & -A-1
\end{array}\right) \quad \text { and } \quad F(z)=\left(\begin{array}{l}
f_{1}(u, v)+u \\
f_{2}(u, v)+v
\end{array}\right)
$$

with

$$
D(Q)=\left\{(u, v) \in D\left((-\Delta)^{\alpha}\right) \times D\left((-\Delta)^{\alpha}\right),\left.\frac{\partial u}{\partial v}\right|_{\partial \Omega}=\left.\frac{\partial v}{\partial v}\right|_{\partial \Omega}=0\right\} .
$$

In the following, we shall prove that system (1.2) has a local solution. Firstly, we present the following lemma.

Lemma 3.1 For very $z_{0} \in X_{+}$, the Cauchy problem (3.1) has a unique maximal local solution

$$
z \in C\left(\left[0, T_{\max }\right) ; X\right) \cap C^{1}\left(\left[0, T_{\max }\right) ; X\right) \cap C\left(\left[0, T_{\max }\right) ; D(A)\right)
$$

which satisfies the following Duhamel formula for $t \in\left[0, T_{\max }\right]$ :

$$
z(t)=e^{t \widetilde{Q}_{z_{0}}}+\int_{0}^{t} e^{(t-s)} \widetilde{Q} F[z(s)] d s
$$

where $\widetilde{Q}$ is an operator and the closure of $Q$ in $X$. Moreover, if $T_{\max }<\infty$, then $\lim \sup _{T_{\max }^{-}}\|z(t)\|=\infty$. 
Proof The operator $\widetilde{Q}$ can generate an analytic, condensed, strong continuous operator semigroup $e^{t \widetilde{Q}}$ in $X$ since it is the closure of $Q$. Furthermore, for $t>0$, we have

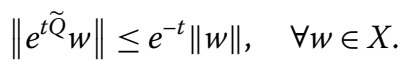

Moreover, one can easily check that $F: D(Q) \rightarrow X$ is locally Lipschitz on a bounded set. Using Theorem 7.2.1 in [18], we obtain that (3.1) has a unique maximal local solution.

Lemma 3.2 The solution $(u(t, x), v(t, x))$ of the initial-boundary problem (1.2) is nonnegative.

Proof We first consider the following system:

$$
\begin{aligned}
& \frac{\partial u^{\prime}}{\partial t}=-(-\Delta)^{\alpha} u^{\prime}+u_{+}^{\prime}\left[-a+e c v_{+}^{\prime} /\left(1+b v_{+}^{\prime}\right)\right] \quad \text { in }(0, T) \times \Omega, \\
& \frac{\partial v_{+}^{\prime}}{\partial t}=-(-\Delta)^{\alpha} v^{\prime}+v_{+}^{\prime}\left[r\left(1-v_{+}^{\prime} / K\right)-c u_{+}^{\prime} /\left(1+b v_{+}^{\prime}\right)\right] \quad \text { in }(0, T) \times \Omega, \\
& \frac{\partial u^{\prime}}{\partial v}=\frac{\partial v^{\prime}}{\partial v}=0 \quad \text { on }(0, T) \times \partial \Omega, \\
& \left(u^{\prime}(0, x), v^{\prime}(0, x)\right)=\left(u_{0}(x), v_{0}(x)\right) \geq(0,0) \quad \text { in } \Omega .
\end{aligned}
$$

Multiplying (3.4) by $u_{-}^{\prime}$ and $v_{-}^{\prime}$, respectively, and integrating on $\Omega$, we have

$$
\begin{aligned}
& -\frac{1}{2} \frac{d}{d t} \int_{\Omega}\left|u^{\prime}\right|^{2} d x=\int_{\Omega} u^{\prime}(-\Delta)^{\alpha} u^{\prime} d x, \\
& -\frac{1}{2} \frac{d}{d t} \int_{\Omega}\left|v_{-}^{\prime}\right|^{2} d x=\int_{\Omega} v_{-}^{\prime}(-\Delta)^{\alpha} v_{-}^{\prime} d x .
\end{aligned}
$$

By using (2.3), for $t \in\left(0, T_{\max }\right)$, we have

$$
\left\|u^{\prime}(t, \cdot)\right\|_{2}^{2}+\left\|v^{\prime}(t, \cdot)\right\|_{2}^{2} \leq\left\|u^{\prime}(0, \cdot)\right\|_{2}^{2}+\left\|v^{\prime}(0, \cdot)\right\|_{2}^{2}=0
$$

which derives that

$$
u^{\prime}(t, x) \geq 0, \quad v^{\prime}(t, x) \geq 0 .
$$

It follows that $\left(u^{\prime}(t, x), v^{\prime}(t, x)\right)$ is a solution of (1.2). By using Lemma 3.1, we obtain that

$$
u(t, x)=u^{\prime}(t, x) \geq 0, \quad v^{\prime}(t, x) \geq 0, \quad t \in\left(0, T_{\max }\right) .
$$

Next, we shall prove that there exists a positive global solution for system (1.2). According to Lemmas 3.1 and 3.2, we need to prove that the solution of (1.2) is uniformly bounded, i.e., dissipation. For this end, we first give out the following lemma. 
Lemma 3.3 Assume that $z(t, x)$ satisfies the following system:

$$
\begin{aligned}
& \frac{\partial z}{\partial t}=-(-\Delta)^{\alpha} z+z(r-z) \quad \text { in }(0, T) \times \Omega, \\
& \frac{\partial z}{\partial v}=0 \quad \text { in }(0, T) \times \Omega, \\
& z(x, 0)=z_{0}(x) \geq 0, \quad \text { in } \Omega .
\end{aligned}
$$

Then $\lim _{t \rightarrow \infty} z(t, x)=r$ for any $x \in \Omega$.

This lemma is easy to prove by using the definition of $(-\Delta)^{\alpha} u$ and inequalities of Strook and Varopoulos, so we omit it.

Remark 3.1 Suppose that $z(t, x)$ satisfies the following equation:

$$
\begin{aligned}
& \frac{\partial z}{\partial t}=-(-\Delta)^{\alpha} z+(r-z) \quad \text { in }(0, T) \times \Omega, \\
& \frac{\partial z}{\partial v}=0 \quad \text { in }(0, T) \times \Omega, \\
& z(x, 0)=z_{0}(x) \geq 0, \quad \text { in } \Omega .
\end{aligned}
$$

Then $\lim _{t \rightarrow \infty} z(t, x)=r$ for any $x \in \Omega$.

Proof It is obvious that system (3.7) always has a constant solution $r$. Let $z$ be the positive solution of (3.7), and define the Lyapunov function $V(z)=\int_{\Omega}[z-r-r \ln (z / r)] d x$, then

$$
\begin{aligned}
\frac{d V(z)}{d t}= & -\int_{\Omega}\left[\left(1-\frac{r}{z}\right)(-\Delta)^{\alpha} z\right] d x-\int_{\Omega}\left(1-\frac{r}{z}\right)(z-r) d x \\
= & -\int_{\Omega}(-\Delta)^{\alpha / 2}\left(1-\frac{r}{z}\right)(-\Delta)^{\alpha / 2} z d x-\int_{\Omega} \frac{1}{z}(r-z)^{2} d x \\
= & -r \int_{\Omega}\left[\left(\text { p.v. } \int_{\Omega} \frac{z(x)-z(y)}{|x-y|^{N+\alpha}} d y\right) \times\left(\text { p.v. } \int_{\Omega} \frac{z(x)-z(y)}{z(x) z(y)|x-y|^{N+\alpha}}\right) d y\right] d x \\
& -\int_{\Omega} \frac{1}{z}(r-z)^{2} d x \\
\leq & 0 .
\end{aligned}
$$

Therefore, $z=r$ is globally asymptotically stable.

Lemma 3.4 Assume that $(u, v)$ is the solution of $(1.2)$, then

$$
\limsup _{t \rightarrow \infty} \max _{x \in \Omega} u(t, x) \leq e\left(\frac{r}{a}+1\right) K, \quad \limsup _{t \rightarrow \infty} \max _{x \in \Omega} v(t, x) \leq K
$$


Proof Using the second equation of model (1.2), we obtain

$$
\begin{aligned}
& \frac{\partial v}{\partial t} \leq-(-\Delta)^{\alpha} v+r(1-v / K) v \quad \text { in }(0, T) \times \Omega, \\
& \frac{\partial v}{\partial v}=0 \quad \text { on }(0, T) \times \partial \Omega \\
& v(0, x)=v_{0}(x) \quad \text { in } \Omega .
\end{aligned}
$$

By using comparison theory and Lemma 3.3, one can easily obtain that

$$
\limsup _{t \rightarrow \infty} \max _{x \in \Omega} v(t, x) \leq K
$$

Multiplying the second equation by $e$ and adding it to the first equation in (1.2), we have

$$
\begin{aligned}
& \frac{\partial \omega}{\partial t} \leq(-\Delta)^{\alpha} \omega+e r(1-v / K) v-a u \quad \text { in }(0, T) \times \Omega, \\
& \frac{\partial \omega}{\partial v}=0 \quad \text { on }(0, T) \times \partial \Omega, \\
& \omega(0, x)=\omega_{0}(x) \quad \text { in } \Omega,
\end{aligned}
$$

where $\omega=u+e v$. Since

$$
e r(1-v / K) v-a u=e(r+a) v-e r v^{2} / K-a \omega \leq e(r+a)(K+\epsilon)-a \omega
$$

in $[T, \infty] \times \Omega$. Therefore, by using Lemma 3.3 , we obtain

$$
\limsup _{t \rightarrow \infty} \max _{x \in \Omega} u(t, x) \leq e(r / a+1)(K+\epsilon)
$$

on $\bar{\Omega}$, which implies the second desired assertion by the continuity as $\epsilon \rightarrow 0$.

Now, by Lemmas 3.1, 3.2 and 3.4, we have the following theorem.

Theorem 3.2 System (1.2) has a unique, non-negative and bounded solution $(u(t, x), v(t, x))$ such that

$$
(u(t, x), v(t, x)) \in C([0, \infty), X) \cap C^{1}((0, \infty), X) \cap C((0, \infty), D(Q)) .
$$

\section{Stability of the equilibria}

In this section we shall prove the stability of the positive constant solution to model (1.2). Note that (1.2) has the following three non-negative constant solutions:

(i) the trivial solution $(0,0)$;

(ii) the semi-trivial solution $(0, K)$;

(iii) the unique positive constant solution $\mathbf{w}_{*}=:\left(u_{*}, v_{*}\right)$, where

$$
u_{*}=\frac{e r}{K(e c-a b)^{2}}[K(e c-a b)-a], \quad v_{*}=\frac{a}{e c-a b} .
$$


For the existence of positive constant solution $\mathbf{w}_{*}$, it is necessary to assume that

$$
0<\frac{a}{K(e c-a b)}<1
$$

Let $E\left(\mu_{i}^{\alpha}\right)(i=1,2,3, \ldots)$ be the eigenvalue space corresponding to $\mu_{i}^{\alpha}$ in $C^{1}(\Omega)$, and $\varphi$ is the set of eigenfunctions corresponding to $\mu^{\alpha}$.

Notation 4.1 (i) $\mathbf{X}_{i j}:=\left\{\mathbf{C} \varphi_{i j}: \mathbf{C} \in R^{2}\right\}$, where $\left\{\varphi_{i j}\right\}$ is the orthonormal basis of $S\left(\mu_{i}^{\alpha}\right)$ for $j=1, \ldots, \operatorname{dim}\left[S\left(\mu_{i}^{\alpha}\right)\right]$.

(ii) $\mathbf{X}:=\left\{(u, v) \in H^{\alpha}(\bar{\Omega}) \times H^{\alpha}(\bar{\Omega}): \frac{\partial u}{\partial v}=\frac{\partial v}{\partial v}\right.$ on $\left.\partial \Omega\right\}$, so that

$$
\mathbf{X}=\bigoplus_{i=1}^{\infty} \bigoplus_{j=1}^{\operatorname{dim}\left[S\left(\mu_{i}\right)\right]} \mathbf{X}_{i j}
$$

The following theorem shows that the positive constant $\mathbf{w}_{*}$ of (1.2) is locally asymptotically stable under certain condition.

Theorem 4.2 If $\frac{b}{e c}+\frac{1}{e c K}<\frac{2}{K(e c-a b)}$, then the positive constant solution $\mathbf{w}_{*}$ of $(1.2)$ is locally asymptotically stable.

Proof The linearized problem of $(1.2)$ at $\left(u_{*}, v_{*}\right)$ can be expressed by

$$
\mathbf{w}_{t}=\left(D+F_{\mathbf{w}}\left(\mathbf{w}_{*}\right)\right) \mathbf{w}
$$

where

$$
\begin{aligned}
& \mathbf{w}=(u(t, x), v(t, x))^{T}, \quad \mathbf{w}_{*}=\left(u_{*}(t, x), v_{*}(t, x)\right)^{T}, \\
& F=\left(-a u+\frac{e c u v}{1+b v}, r\left(1-\frac{v}{K}\right) v-\frac{c u v}{1+b v}\right), \\
& F_{\mathbf{w}}\left(\mathbf{w}_{*}\right)=\left(\begin{array}{cc}
0 & \frac{e c u_{*}}{\left(1+b v_{*}\right)^{2}} \\
-\frac{c v_{*}}{1+b v_{*}} & r-\frac{2 r}{K} \nu_{*}-\frac{c u_{*}}{\left(1+b v_{*}\right)^{2}}
\end{array}\right),
\end{aligned}
$$

and

$$
D=\left(\begin{array}{cc}
-(-\Delta)^{\alpha} & 0 \\
0 & -(-\Delta)^{\alpha}
\end{array}\right) .
$$

Consider the following eigenvalue problem:

$$
\left(D+F_{\mathbf{w}}\left(\mathbf{w}_{*}\right)\right)\left(\begin{array}{l}
\phi \\
\psi
\end{array}\right)=\lambda\left(\begin{array}{l}
\phi \\
\psi
\end{array}\right) .
$$

$\lambda$ is an eigenvalue of $D+F_{\mathbf{w}}\left(\mathbf{w}_{*}\right)$ if and only if $\lambda$ is an eigenvalue of the matrix $-\mu_{k}^{\alpha} I+$ $F_{\mathbf{w}}\left(\mathbf{w}_{*}\right)$ for each $k \geq 1$. Therefore, to study the local stability at $\mathbf{w}_{*}$, it is necessary to investigate the characteristic polynomial

$$
\operatorname{det}\left(\lambda I+\mu_{k}^{\alpha} I-F_{\mathbf{w}}\left(\mathbf{w}_{*}\right)\right)=\lambda^{2}+B_{1} \lambda+B_{2}
$$


where

$$
\begin{aligned}
& B_{1}=2 \mu_{k}^{\alpha}-\left(r-\frac{2 r}{K} \nu_{*}-\frac{c u_{*}}{\left(1+b v_{*}\right)^{2}}\right), \\
& B_{2}=\frac{e c^{2} u_{*} \nu_{*}}{\left.1+b v_{*}\right)^{3}}+\mu_{k}^{\alpha}\left(\mu_{k}^{\alpha}-\left(r-\frac{2 r}{K} \nu_{*}-\frac{c u_{*}}{\left(1+b v_{*}\right)^{2}}\right)\right) .
\end{aligned}
$$

Using the condition of Theorem 4.2, we obtain

$$
r-\frac{2 r}{K} v_{*}-\frac{c u_{*}}{\left(1+b v_{*}\right)^{2}}=r a\left(\frac{b}{e c}+\frac{1}{e c K}-\frac{2}{K(e c-a b)}\right)<0 .
$$

The two eigenvalues of $D+F_{\mathbf{w}}\left(\mathbf{w}_{*}\right)$ have negative real parts. Therefore the positive constant solution $\mathbf{w}_{*}$ of (1.2) is locally asymptotically stable, and thus no bifurcation occurs.

The following theorem is the global stability result of the positive constant solution $\mathbf{w}_{*}$.

Theorem 4.3 Assume that $b K \leq \frac{e c}{e c-a b}$, then the positive constant solution $\mathbf{w}_{*}=\left(u_{*}, v_{*}\right)$ of (1.2) is globally asymptotically stable.

Proof . Let $(u(x, t), v(x, t))$ be a positive solution of (1.2) and define the following Lyapunov function:

$$
E(t)=\int_{\Omega}\left[h\left(u-u_{*}-u_{*} \ln \frac{u}{u_{*}}\right)+\left(v-v_{*}-v_{*} \ln \frac{v}{v_{*}}\right)\right] d x,
$$

where $h=: \frac{1+b v_{*}}{e}>0$. Then we have

$$
\begin{aligned}
E^{\prime}(t)= & \int_{\Omega}\left[h\left(1-\frac{u_{*}}{u}\right) u_{t}+\left(1-\frac{v_{*}}{v}\right) v_{t}\right] d x, \\
E^{\prime}(t)= & E_{1}(t)+\int_{\Omega} h\left(u-u_{*}\right)\left(-a+\frac{e c v}{1+b v}\right) d x+\int_{\Omega}\left(v-v_{*}\right)\left(r-\frac{r v}{K}-\frac{c u}{1+b v}\right) d x \\
= & E_{1}(t)+\int_{\Omega} h\left(u-u_{*}\right)\left(-a+\frac{e c v}{1+b v}+a-\frac{e c v_{*}}{1+b v_{*}}\right) d x \\
& +\int_{\Omega}\left(v-v_{*}\right)\left(r-\frac{r v}{K}-\frac{c u}{1+b v}-r+\frac{r v_{*}}{K}+\frac{c u_{*}}{1+b v_{*}}\right) d x \\
= & E_{1}(t)+\int_{\Omega} \frac{\left(u-u_{*}\right)\left(v-v_{*}\right)}{(1+b v)\left(1+b v_{*}\right)}\left[-c\left(1+b v_{*}-e h\right)\right] d x \\
& +\int_{\Omega}\left(v-v_{*}\right)^{2}\left(-\frac{r}{K}+\frac{c b u_{*}}{(1+b v)\left(1+b v_{*}\right)}\right) d x \\
\leq & E_{1}(t)+\int_{\Omega}\left(v-v_{*}\right)^{2}\left(-\frac{r}{K}+\frac{c b u_{*}}{(1+b v)\left(1+b v_{*}\right)}\right) d x,
\end{aligned}
$$

where

$$
\begin{aligned}
E_{1}(t) & =-h \int_{\Omega}\left[\left(1-\frac{u_{*}}{u}\right)(-\Delta)^{\alpha} u\right] d x-\int_{\Omega}\left[\left(1-\frac{v_{*}}{v}\right)(-\Delta)^{\alpha} v\right] d x \\
& =-h \int_{\Omega}(-\Delta)^{\alpha / 2}\left(1-\frac{u_{*}}{u}\right)(-\Delta)^{\alpha / 2} u d x-\int_{\Omega}(-\Delta)^{\alpha / 2}\left(1-\frac{\nu_{*}}{v}\right)(-\Delta)^{\alpha / 2} v d x
\end{aligned}
$$




$$
\begin{aligned}
= & -h u^{*} \int_{\Omega}\left[\left(\text { p.v. } \int_{\Omega} \frac{u(x)-u(y)}{|x-y|^{N+\alpha}} d y\right) \times\left(\text { p.v. } \int_{\Omega} \frac{u(x)-u(y)}{u(x) u(y)|x-y|^{N+\alpha}} d y\right)\right] d x \\
& -v^{*} \int_{\Omega}\left[\left(\text { p.v. } \int_{\Omega} \frac{v(x)-v(y)}{|x-y|^{N+\alpha}} d y\right) \times\left(\text { p.v. } \int_{\Omega} \frac{v(x)-v(y)}{v(x) v(y)|x-y|^{N+\alpha}} d y\right)\right] d x \\
\leq & 0 .
\end{aligned}
$$

In the above derivation, note that $-a+\frac{e c v_{*}}{1+b v_{*}}=0$ and $r-\frac{r v_{*}}{K}-\frac{c u_{*}}{1+b v_{*}}=0$.

$$
\begin{aligned}
-\frac{r}{K}+\frac{c b u_{*}}{(1+b v)\left(1+b v_{*}\right)} & <-\frac{r}{K}+\frac{c b u_{*}}{1+b v_{*}} \\
& =-\frac{r}{K}+c b u_{*} \cdot \frac{e c-a b}{e c} \\
& =-\frac{r}{K}+\frac{b r}{K(e c-a b)}[K(e c-a b)-a] \\
& =-\frac{r}{K}\left(1-b K+\frac{a b}{e c-a b}\right) \leq 0,
\end{aligned}
$$

and thus $E^{\prime}(t) \leq 0$, which implies the desired assertion since the equality holds only when $(u, v)=\left(u_{*}, v_{*}\right)$. By the regular argument, one can derive that $\mathbf{w}_{*}=\left(u_{*}, v_{*}\right)$ of $(1.2)$ is globally asymptotically stable.

Remark 4.4 Assume that $b K \leq \frac{e c}{e c-a b}$, then the positive constant solution $\mathbf{w}_{*}=\left(u_{*}, v_{*}\right)$ is globally asymptotically stable to (1.2), that is to say, model (1.2) does not have non-constant positive steady states.

\section{Remark 4.5 If}

$$
\frac{b}{e c}+\frac{1}{e c K}>\frac{2}{K(e c-a b)}
$$

then, in view of Theorem 4.2, there may exist non-constant positive steady states to model (1.2).

Remark 4.6 Using the condition $b K<\frac{e c}{e c-a b}$ of Theorem 4.3, we can easily obtain that the inequality $\frac{b}{e c}+\frac{1}{e c K}<\frac{2}{K(e c-a b)}$ holds.

\section{The existence of non-constant positive steady states}

In this section, we shall investigate the existence of non-constant positive solution for (5.1).

\subsection{A priori estimates of positive solution}

The corresponding steady-state system of (1.2) is as the following fractional diffusion system:

$$
\begin{aligned}
& (-\Delta)^{\alpha} u=-a u+e c u v /(1+b v) \quad x \in \Omega, \\
& (-\Delta)^{\alpha} v=r(1-v / K) v-c u v /(1+b v) \quad x \in \Omega, \\
& \frac{\partial u}{\partial v}=\frac{\partial v}{\partial v}=0 \quad x \in \partial \Omega .
\end{aligned}
$$


In the following, the generic constants $A_{1}, A_{1}^{*}$ and $A_{2}$ will depend on the domain $\Omega$.

The main purpose of this subsection is to give a priori positive upper and lower bounds for the positive solutions to system (5.1). To this goal, we shall make use of the following two propositions.

Proposition 5.1 (Harnack's inequality $[19,20])$ Let $c(x) \in C(\bar{\Omega})$ and $w(x)$ be a positive solution to $(-\Delta)^{\alpha} w(x)+c(x) w(x)=0$ in $\Omega$. Then there exists a positive constant $C_{*}$ which depends only on $\alpha$, dimension and $c(x)$ such that

$$
\max _{\bar{\Omega}} w \leq C_{*} \min _{\bar{\Omega}} w
$$

Proposition 5.2 (Maximum principle) Let $g \in C(\Omega \times R)$.

(i) If $w \in C(\Omega)$ satisfies $(-\Delta)^{\alpha} w(x)+g(x, w(x)) \geq 0$ in $\Omega$, and $w\left(x_{0}\right)=\min _{\bar{\Omega}} w$, then $g\left(x_{0}, w\left(x_{0}\right)\right) \geq 0$.

(ii) If $w \in C$ satisfies $(-\Delta)^{\alpha} w(x)+g(x, w(x)) \leq 0$ in $\Omega$, and $w\left(x_{0}\right)=\max _{\bar{\Omega}} w$, then $g\left(x_{0}, w\left(x_{0}\right)\right) \leq 0$.

Proof By using the definition of the fractional Laplacian operator (2.2), one can easily obtain these results.

Lemma 5.1 The positive solution of (5.1) satisfies

$$
\max _{x \in \Omega} u(x) \leq e\left(\frac{r}{a}+1\right) K, \quad \max _{x \in \Omega} v(x) \leq K
$$

Proof By using the comparison argument to the second equation of (5.1), one can easily obtain $\max _{x \in \Omega} v(x) \leq K$. Multiplying the second equation by $e$ and adding it to the first equation in (5.1), then using the comparison argument, we obtain $\max _{x \in \Omega} u(x) \leq e\left(\frac{r}{a}+\right.$ 1)K.

Lemma 5.2 For any positive solution $(u, v)$ of $(5.1)$, there exists a positive constant $\underline{C}$ such that

$$
\min _{x \in \Omega} u(x) \geq \underline{C}, \quad \min _{x \in \Omega} v(x) \geq \underline{C} .
$$

Proof Multiplying the first equation of (5.1) by $u$ and integrating on $\Omega$, by (2.3), we get $\int_{\Omega} u^{2}\left(-a+\frac{e c v}{1+b v}\right) d x \geq 0$. Therefore, there exists $y_{2} \in \Omega$ such that $-a-a b v\left(y_{2}\right)+e c v\left(y_{2}\right) \geq 0$, which implies that $v\left(y_{2}\right) \geq \frac{a}{e c}$. This, combined with $\frac{\max _{\bar{\Omega}} v}{\min _{\bar{\Omega}} v} \leq A$, yields that

$$
\min _{\bar{\Omega}} v \geq \frac{\max _{\bar{\Omega}} v}{A_{1}} \geq \frac{v\left(y_{2}\right)}{A_{1}} \geq \frac{a}{e c A_{1}}>0
$$

Now, we prove that $\min _{\bar{\Omega}} u(x)>\underline{C}$. Assume on the contrary that there exists a solution sequence $\mathbf{w}_{m}=\left(u_{m}, v_{m}\right)$ to (5.1) satisfying

$$
\lim _{m \rightarrow \infty} \min _{\bar{\Omega}} u_{m}=0 .
$$


Using the first equation of system (5.1) and Harnack's inequality, we obtain $\max _{\bar{\Omega}} u_{m} \leq$ $A_{2} \min _{\bar{\Omega}} u_{m}$. Therefore we have $\max _{\bar{\Omega}} u_{m} \rightarrow 0$ and $u_{m} \rightarrow 0$ uniformly as $m \rightarrow \infty$.

We may assume, by passing to a subsequence if necessary, that as $m \rightarrow \infty,\left(u_{m}, v_{m}\right) \rightarrow$ $(u, v)$, where $u$ and $v$ are non-negative functions.

Therefore, by passing to a subsequence if necessary, we obtain that $v$ satisfies

$$
\begin{aligned}
& (-\Delta)^{\alpha} v=r(1-v / K) v \quad x \in \Omega \\
& \frac{\partial v}{\partial v}=0 \quad x \in \partial \Omega
\end{aligned}
$$

Using the maximum principle, we have $v \equiv K$. Let the first equation to (5.1) be divided by $\max _{\bar{\Omega}} u_{m}$. Then, by a similar argument as that in (5.4), we get $\frac{u_{m}}{\max _{\bar{\Omega}} u_{m}} \rightarrow \bar{u}$ uniformly. Combined with $\frac{u_{m}}{\max _{\bar{\Omega}} u_{m}} \geq \frac{\min _{\bar{\Omega}} u_{m}}{\max \bar{\Omega}_{\bar{\Omega}} u_{m}} \geq \frac{1}{A_{1}^{*}}$, this yields that $\bar{u} \geq \frac{1}{A_{1}^{*}}$. Then, integrating, we have

$$
\int_{\Omega} \frac{u_{m}}{\max _{\bar{\Omega}} u_{m}}\left(-a+\frac{e c v_{m}}{1+b v_{m}}\right) d x=0
$$

Let $m \rightarrow \infty$, and we get $K=\frac{a}{e c-a b}$, which is a contradiction to $\frac{a}{K(e c-a b)}<1$. Therefore, $\min _{\bar{\Omega}} u(x) \geq \underline{C}$ if $\frac{a}{K(e c-a b)}<1$.

\subsection{The existence of non-constant positive steady states}

By Lemmas 5.1 and 5.2, there exists a positive constant $C$ such that $C^{-1}<u, v<C$. Define

$$
\begin{aligned}
& \mathbf{X}^{+}=\left\{(u, v)^{T} \in \mathbf{X} \mid u>0, v>0, x \in \bar{\Omega}\right\}, \\
& B(C)=\left\{(u, v)^{T} \in \mathbf{X} \mid C^{-1}<u, v<C, x \in \bar{\Omega}\right\},
\end{aligned}
$$

then $\mathbf{X}=\bigoplus_{i=1}^{\infty} \mathbf{X}_{i}$, where $\mathbf{X}_{i}=\bigoplus_{j=1}^{\operatorname{dim} E\left(\mu_{i}\right)} \mathbf{X}_{i j}$. Employing the notation in Section 4, (5.1) can be written as

$$
\begin{aligned}
& (-\Delta)^{\alpha} \mathbf{w}=\mathbf{F}(\mathbf{w}) \text { in } \Omega, \\
& \frac{\partial \mathbf{w}}{\partial v}=0 \quad \text { on } \partial \Omega .
\end{aligned}
$$

Therefore $\mathbf{w}$ is a positive solution of (5.5) if and only if

$$
\mathbf{G}(\mathbf{w}) \triangleq \mathbf{w}-(\mathbf{I}-D)^{-1}[\mathbf{F}(\mathbf{w})+\mathbf{w}]=0, \quad \mathbf{w} \in \mathbf{X}^{+},
$$

where $(\mathbf{I}-D)^{-1}$ is the inverse of $\mathbf{I}-D$ in $\mathbf{X}$. As $\mathbf{G}(\cdot)$ is a compact perturbation of the identity operator by Lemma 2.1 , for any $B \triangleq B(C)$, the Leray-Schauder degree $\operatorname{deg}(\mathbf{G}(\cdot), \mathbf{0}, \mathbf{B})$ is well defined if $\mathbf{G}(\mathbf{w}) \neq 0$ on $\partial \mathbf{B}$. Moreover, we note that

$$
D_{\mathbf{w}} \mathbf{G}\left(\mathbf{w}_{*}\right)=\mathbf{I}-(\mathbf{I}-D)^{-1}\left\{\mathbf{F}_{\mathbf{w}}\left(\mathbf{w}_{*}\right)+\mathbf{I}\right\},
$$

where $\mathbf{w}_{*}=\left(u_{*}, v_{*}\right)^{T}$. We recall that if $D_{\mathbf{w}} \mathbf{G}\left(\mathbf{w}_{*}\right)$ is invertible, the index of $\mathbf{G}$ at $\mathbf{w}_{*}$ is defined as the index $\left(\mathbf{G}(\cdot), \mathbf{w}_{*}\right)=(-1)^{\gamma}$, where $\gamma$ is the number of negative eigenvalues of $D_{\mathbf{w}} \mathbf{G}\left(\mathbf{w}_{*}\right)$. 
We note that for each integer $i \geq 1$ and each integer $1 \leq j \leq \operatorname{dim} E\left(\mu_{i}^{\alpha}\right), \mathbf{X}_{i j}$ is invariant under $D_{\mathbf{w}} \mathbf{G}\left(\mathbf{w}_{*}\right)$, namely $D_{\mathbf{w}} \mathbf{G}\left(\mathbf{w}_{*}\right) \mathbf{w} \in \mathbf{X}_{i j}$ for all $\mathbf{w} \in \mathbf{X}_{i j}$. Thus, $\lambda$ is an eigenvalue of $D_{\mathbf{w}} \mathbf{G}\left(\mathbf{w}_{*}\right)$ on $\mathbf{X}_{i j}$ if and only if it is an eigenvalue of the matrix

$$
\mathbf{I}-\frac{1}{1+\mu_{i}^{\alpha}}\left\{\mathbf{F}_{\mathbf{w}}\left(\mathbf{w}_{*}\right)+\mathbf{I}\right\}=\frac{1}{1+\mu_{i}^{\alpha}}\left\{\mu_{i}^{\alpha} \mathbf{I}-\mathbf{F}_{\mathbf{w}}\left(\mathbf{w}_{*}\right)\right\} .
$$

Thus, $D_{\mathbf{w}} \mathbf{G}\left(\mathbf{w}_{*}\right)$ is invertible if and only if, for all $i \geq 1$, the matrix

$$
\mathbf{I}-\frac{1}{1+\mu_{i}^{\alpha}}\left\{\mathbf{F}_{\mathbf{w}}\left(\mathbf{w}_{*}\right)+\mathbf{I}\right\}
$$

is non-singular. Writing

$$
H\left(\mu^{\alpha}\right) \triangleq H\left(\mathbf{w}_{*}, \mu^{\alpha}\right)=\operatorname{det}\left[\mu^{\alpha} \mathbf{I}-\mathbf{F}_{\mathbf{w}}\left(\mathbf{w}_{*}\right)\right]
$$

furthermore, we note that the sign of $\operatorname{det}\left\{\mathbf{I}-\frac{1}{1+\mu_{i}^{\alpha}}\left[\mathbf{F}_{\mathbf{w}}\left(\mathbf{w}_{*}\right)+\mathbf{I}\right]\right\}$ depends on the number of negative eigenvalues of $\mathbf{I}-\frac{1}{1+\mu_{i}^{\alpha}}\left[\mathbf{F}_{\mathbf{w}}\left(\mathbf{w}_{*}\right)+\mathbf{I}\right]$, and both $H\left(\mu_{i}^{\alpha}\right)$ and $\operatorname{det}\left\{\mathbf{I}-\frac{1}{1+\mu_{i}^{\alpha}}\left[\mathbf{F}_{\mathbf{w}}\left(\mathbf{w}_{*}\right)+\mathbf{I}\right]\right\}$ have the same signs. Therefore, if $H\left(\mu_{i}^{\alpha}\right) \neq 0$, then for each integer $1 \leq j \leq \operatorname{dim} E\left(\mu_{i}^{\alpha}\right)$, the number of negative eigenvalues of $D_{\mathbf{w}} \mathbf{G}\left(\mathbf{w}_{*}\right)$ on $\mathbf{X}_{i j}$ is odd if and only if $H\left(\mu_{i}^{\alpha}\right)<0$.

In conclusion, we have the following.

Lemma 5.3 Suppose that, for all $i \geq 1$, the matrix $\mu_{i}^{\alpha} \mathbf{I}-\mathbf{F}_{\mathbf{w}}\left(\mathbf{w}_{*}\right)$ is non-singular. Then

$$
\operatorname{index}\left(\mathbf{G}(\cdot), \mathbf{w}_{*}\right)=(-1)^{\gamma}, \quad \text { where } \gamma=\sum_{i \geq 1, H\left(\mu_{i}^{\alpha}\right)<0} \operatorname{dim} \mathbf{E}\left(\mu_{i}^{\alpha}\right) \text {. }
$$

To facilitate our computation of index $\left(\mathbf{G}(\cdot), \mathbf{w}_{*}\right)$, we shall consider carefully the sign of $H\left(\mu_{i}\right)$. Note that

$$
H\left(\mu^{\alpha}\right)=\operatorname{det}\left[\mu^{\alpha} \mathbf{I}-\mathbf{F}_{\mathbf{w}}\left(\mathbf{w}_{*}\right)\right]
$$

so we shall only need to consider $\operatorname{det}\left[\mu^{\alpha} \mathbf{I}-\mathbf{F}_{\mathbf{w}}\left(\mathbf{w}_{*}\right)\right]$.

In the following, by analyzing the two roots of $H\left(\mu^{\alpha}\right)=0$, we discuss the sign of $H\left(\mu_{i}^{\alpha}\right)$. As

$$
F_{\mathbf{w}}\left(\mathbf{w}_{*}\right)=\left(\begin{array}{cc}
0 & \frac{e c u_{*}}{\left(1+b b_{*}\right)^{2}} \\
-\frac{c v_{*}}{1+b v_{*}} & r-\frac{2 r}{K} v_{*}-\frac{c u_{*}}{\left(1+b v_{*}\right)^{2}}
\end{array}\right),
$$

we obtain

$$
\operatorname{det}\left\{\mu^{\alpha} \mathbf{I}-\mathbf{F}_{\mathbf{w}}\left(\mathbf{w}_{*}\right)\right\}=\mu^{2 \alpha}+\widetilde{B} \mu^{\alpha}+\widetilde{C},
$$

where

$$
\widetilde{B}=-\left(r-\frac{2 r}{K} \nu_{*}-\frac{c u_{*}}{\left(1+b v_{*}\right)^{2}}\right), \quad \widetilde{C}=\frac{e c^{2} u_{*} v_{*}}{\left(1+b v_{*}\right)^{3}}>0 .
$$


When $\widetilde{B}^{2}-4 \widetilde{C}>0$, let $\widetilde{\mu}_{1}$ and $\widetilde{\mu}_{2}$ be two of $\operatorname{det}\left\{\mu^{\alpha} \mathbf{I}-\mathbf{F}_{\mathbf{w}}\left(\mathbf{w}_{*}\right)\right\}=0$ with $\widetilde{\mu}_{1}(d)<\widetilde{\mu}_{2}(d)$. Therefore, if $\widetilde{B}>0, \widetilde{\mu}_{1}$ and $\tilde{\mu}_{2}$ are non-positive; whereas if both $\tilde{\mu}_{1}$ and $\widetilde{\mu}_{2}$ are positive and $\widetilde{\mu}_{1} \neq \widetilde{\mu}_{2}$, then it is necessary that $\widetilde{B}<0$ and $\widetilde{B}^{2}-4 \widetilde{C}>0$.

Lemma 5.4 Suppose that $\frac{b}{e c}+\frac{1}{e c K}>\frac{2}{K(e c-a b)}$ and $\widetilde{B}^{2}-4 \widetilde{C}>0$, then there exist positive integers $k_{1}$ and $k_{2}$ such that $\mu_{k_{1}}^{\alpha} \leq \widetilde{\mu}_{1}<\mu_{k_{1}+1}^{\alpha}$ and $\mu_{k_{2}}^{\alpha}<\tilde{\mu}_{2}<\mu_{k_{2}+1}^{\alpha}$.

To sum up, we obtain the following.

Lemma 5.5 If $0<\widetilde{\mu}_{1}<\mu_{i}^{\alpha}<\widetilde{\mu}_{2}$ for some $i \geq 1$, then $H\left(\mu_{i}^{\alpha}\right)<0$; whereas $H\left(\mu_{i}^{\alpha}\right)>0$ provided $\mu_{i}^{\alpha}<\tilde{\mu}_{1}$ or $\mu_{i}^{\alpha}>\tilde{\mu}_{2}$ for some $i \geq 1$.

Next, we shall discuss the global existence of non-constant positive solution (1.2) with respect to fractional diffusion, as other parameters $r, e, c, b$ and $K$ are fixed. Our results are as follows.

Theorem 5.3 Let the parameters $r, e, c, b, K$ satisfy $\frac{b}{e c}+\frac{1}{e c K}>\frac{2}{K(e c-a b)}$ and $\widetilde{B}^{2}-4 \widetilde{C}>0$. If there exist $k_{1} \geq 1$ and $k_{2} \geq k_{1}$ such that the sum $\delta_{n}=\sum_{i=k_{1}+1}^{k_{2}} \operatorname{dim} E\left(\mu_{i}^{\alpha}\right)$ is odd, then (5.5) has at least one non-constant positive solution.

Proof The proof, which is by contradiction, is based on the homotopy invariance of the topological degree. Suppose on the contrary that the assertion is not true.

Let $d$ be a positive constant which is large enough such that

$$
\frac{-\widetilde{B} d+\sqrt{d^{2} \widetilde{B}^{2}-4 d^{2} \widetilde{C}}}{2 d^{2}}<\mu_{2}^{\alpha}
$$

For $t \in[0,1]$, define

$$
\mathfrak{D}(t)=\left(\begin{array}{cc}
t+(1-t) d & 0 \\
0 & t+(1-t) d
\end{array}\right),
$$

and consider the problem

$$
\begin{aligned}
& -D \mathbf{w}=\mathfrak{D}^{-1}(t) \mathbf{F}(\mathbf{w}) \quad \text { in } \Omega, \\
& \frac{\partial \mathbf{w}}{\partial v}=0 \quad \text { on } \partial \Omega .
\end{aligned}
$$

Then $\mathbf{w}$ is a non-constant positive solution of problem (5.5) if and only if it is a solution of (5.8) for $t=1$. It is obvious that $\mathbf{w}_{*}$ is the unique constant positive solution of (5.8) for any $0 \leq t \leq 1$. For any $0 \leq t \leq 1, \mathbf{w}$ is a positive solution of (5.8) if and only if

$$
\mathbf{G}(t ; \mathbf{w}) \triangleq \mathbf{w}-(\mathbf{I}-D)^{-1}\left\{\mathfrak{D}(t)^{-1} \mathbf{F}(\mathbf{w})+\mathbf{w}\right\}=0, \quad \mathbf{w} \in \mathbf{X}^{+} .
$$

It is obvious that $\mathbf{G}(1 ; \mathbf{w})=\mathbf{G}(\mathbf{w})$. By a direct computation, we obtain

$$
D_{\mathbf{w}} \mathbf{G}\left(t ; \mathbf{w}_{*}\right)=\mathbf{I}-(\mathbf{I}-D)^{-1}\left\{\mathfrak{D}^{-1}(t) \mathbf{F}_{\mathbf{w}}\left(\mathbf{w}_{*}\right)+\mathbf{I}\right\} .
$$


In particular

$$
\begin{aligned}
& D_{\mathbf{w}} \mathbf{G}\left(0 ; \mathbf{w}_{*}\right)=\mathbf{I}-(\mathbf{I}-D)^{-1}\left\{\widetilde{D}^{-1} \mathbf{F}_{\mathbf{w}}\left(\mathbf{w}_{*}\right)+\mathbf{I}\right\}, \\
& D_{\mathbf{w}} \mathbf{G}\left(1 ; \mathbf{w}_{*}\right)=\mathbf{I}-(\mathbf{I}-D)^{-1}\left\{\mathbf{F}_{\mathbf{w}}\left(\mathbf{w}_{*}\right)+\mathbf{I}\right\}=D_{\mathbf{w}} G\left(\mathbf{w}_{*}\right),
\end{aligned}
$$

where

$$
\widetilde{D}=\left(\begin{array}{ll}
d & 0 \\
0 & d
\end{array}\right) .
$$

It is easy to check that, for any $\mu_{i}^{\alpha}$,

$$
H_{0}\left(\mu_{i}^{\alpha}\right)=\operatorname{det}\left\{\widetilde{D}^{-1}\right\} \operatorname{det}\left\{\mu_{i}^{\alpha} \widetilde{D}-\mathbf{F}_{\mathbf{w}}\left(\mathbf{w}_{*}\right)\right\}>0 .
$$

Obviously, zero is not an eigenvalue of the matrix $\mu_{i}^{\alpha} I-\mathbf{F}_{\mathbf{w}}\left(\mathbf{w}_{*}\right)$ for all $i \geq 1$, and

$$
\sum_{i \geq 1, H_{1}\left(\mu_{i}^{\alpha}\right)<0} \operatorname{dim} E\left(\mu_{i}^{\alpha}\right)=\sum_{i=k_{1}+1}^{k_{2}} E\left(\mu_{i}^{\alpha}\right)=\delta_{k},
$$

which is odd. Using Lemma 5.3, we have

$$
\operatorname{index}\left(G(1 ; \cdot), \mathbf{w}_{*}\right)=(-1)^{\gamma}=(-1)^{\delta_{k}}=-1 \text {. }
$$

On the other hand, since $H_{0}\left(\mu_{i}\right)>0$ for all $i \geq 1$, we have

$$
\operatorname{index}\left(G(0 ; \cdot), \mathbf{w}_{*}\right)=(-1)^{0}=1
$$

By Lemmas 5.1 and 5.2, there exists a positive constant $C$ such that, for all $0 \leq t \leq 1$, the positive solutions of (5.8) satisfy $1 / C<u, v<C$. Therefore, $\mathbf{G}(t ; \mathbf{w}) \neq 0$ on $\partial B(C)$ for all $0 \leq t \leq 1$. By the homotopy invariance of the topological degree,

$$
\operatorname{deg}(\mathbf{G}(1 ; \cdot), 0, B(C))=\operatorname{deg}(\mathbf{G}(0 ; \cdot), 0, B(C)) .
$$

Since both equations $\mathbf{G}(1 ; \mathbf{w})=0$ and $\mathbf{G}(0 ; \mathbf{w})=0$ have only the positive solution $\mathbf{w}_{*}$ in $B(C)$, we have

$$
\begin{aligned}
& \operatorname{deg}(\mathbf{G}(0 ; \cdot), 0, B(C))=\operatorname{index}\left(\mathbf{G}(0 ; \cdot), \mathbf{w}_{*}\right)=1, \\
& \operatorname{deg}(\mathbf{G}(1 ; \cdot), 0, B(C))=\operatorname{index}\left(\mathbf{G}(1 ; \cdot), \mathbf{w}_{*}\right)=-1 .
\end{aligned}
$$

This contradiction yields the desired result.

Example 5.4 If we take $a=0.2, e=0.6, c=0.5, K=15, b=0.8, r=3$ and $\alpha=0.5$, by direct computations, then we have $u_{*}=11.63355, v_{*}=1.4286, \widetilde{B}=-1.16196<0, \widetilde{C}=0.253335$, $\widetilde{B}^{2}-4 \widetilde{C}=0.33681>0, \frac{b}{e c}+\frac{1}{e c K}=2.88887, \frac{2}{K(e c-a b)}=0.95238$. Therefore, in some suitable environments, the conditions of Theorem 5.3 are all satisfied, then (5.5) has at least one 
non-constant positive solution, which implies that the predator and the prey can coexist and this gives rise to a spatial pattern.

For a certain sea area in which there are reef islands, the diffusion of the predating fish and the predated fish is fractional diffusion. In this area, if all conditions of Theorem 5.3 are fulfilled for system (5.5), then the predating fish and the predated fish can coexist and multiply.

\section{Competing interests}

The author declares that they have no competing interests.

\section{Acknowledgements}

The author thanks the anonymous referee very much for the valuable comments and suggestions to improve the contents of this article. This work was supported by the National Natural Science Foundation of China (11461023) and the research funds of Ph.D for Honghe University (14bs19).

\section{Publisher's Note}

Springer Nature remains neutral with regard to jurisdictional claims in published maps and institutional affiliations.

Received: 9 November 2016 Accepted: 24 April 2017 Published online: 12 June 2017

\section{References}

1. Chen, WY, Peng, R: Stationary patterns created by cross-diffusion for the competitor-mutualist model. J. Math. Anal. Appl. 291 (2), 550-564 (2004)

2. Hang, PY, Wang, M: Strategy and stationary pattern in a three-species predator-prey model. J. Differ. Equ. 200 245-273 (2004)

3. Ko, W, Ryu, K: Coexistence states of a predator-prey system with non-monotonic functional response. Nonlinear Anal., Real World Appl. 8, 769-786 (2007)

4. Wang, MX: Stationary patterns of strongly coupled prey-predator models. J. Math. Anal. Appl. 292, 484-505 (2004)

5. Ko, W, Ryu, K: A qualitative study on general Gause-type predator-prey models with non-monotonic functional response. Nonlinear Anal., Real World Appl. 10, 2558-2573 (2009)

6. Mumby, PJ, Hastings, A, Edwards, HJ: Thresholds and the resilience of Caribbean coral reefs. Nature 450, 98-101 (2007)

7. Hnaien, D, Kellil, F, Lassoued, R: Asymptotic behavior of global solutions of an anomalous diffusion system. J. Math. Anal. Appl. (2014). doi:10.1016/j.jmaa.2014.07.083

8. Secchi, S: Ground state solutions for nonlinear fractional Schrödinger equations in $R^{N}$. J. Math. Phys. 54 (2013)

9. Shang, X, Zhang, J: Ground states for fractional Schrödinger equations with critical growth. Nonlinearity 27, 187 (2014)

10. Applebaum, D: Lévy Processes and Stochastic Calculus. Cambridge University Press, Cambridge (2009)

11. López-Gómez, J: The steady sates of a non-cooperative model of nuclear reactors. J. Differ. Equ. 246, 358-372 (2009)

12. Ni, WM: Diffusion, cross-diffusion and their spike-layer steady states. Not. Am. Math. Soc. 45(1), 9-18 (1998)

13. Peng, R, Shi, JP: Non-existence of non-constant positive steady-states of two Holling-type-ll predator-prey systems: strong interaction case. J. Differ. Equ. 247, 866-886 (2009)

14. Wang, MX: Non-constant positive steady states of the Sel'kov model. J. Differ. Equ. 190, 600-620 (2003)

15. Letnivov, AV: Theory of differentiation of fractional order. Mat. Sb. 3, 1-7 (1968)

16. Nezza, ED, Palatucci, G, Valdinoci, E: Hitchhiker's guide to the fractional Sobolev space. Bull. Sci. Math. 136, 521-573 (2012)

17. Liskevich, VA, Semenov, YA: Some inequalities for sub-Markovian generators and their applications to the perturbation theory. Proc. Am. Math. Soc. 119, 1171-1177 (1993)

18. Cholewa, JW, Dlotko, T: Global Attractors in Abstract Parabolic Problems, vol. 278. Cambridge University Press, Cambridge (2000)

19. Caffarelli, L, Silvestre, L: An extension problem related to the fractional Laplacian. Commun. Partial Differ. Equ. 32, $1245-1260$ (2007)

20. Stinga, PR, Zhang, C: Harnack's inequality for fractional nonlocal equations (2012), arXiv:1203.1518 